\title{
Double blind, randomised, placebo controlled study of oral vancomycin in prevention of necrotising enterocolitis in preterm, very low birthweight infants
}

\author{
Y K Siu, P C Ng, S C K Fung, C H Lee, M Y Wong, T F Fok, K W So, K L Cheung, \\ W Wong, A F B Cheng
}

\begin{abstract}
Aims-To evaluate the effectiveness of oral vancomycin in the prophylaxis of necrotising enterocolitis in preterm, very low birthweight infants.

Methods-A prospective, double blind, randomised, placebo controlled study in a tertiary referral centre of a university teaching hospital was conducted on 140 very low birthweight infants consecutively admitted to the neonatal unit. The babies were randomly allocated to receive oral vancomycin $(15 \mathrm{mg} / \mathrm{kg}$ every 8 hours for 7 days) or an equivalent volume of placebo solution. Prophylaxis was started 24 hours before the start of oral feeds. All suspected cases of necrotising enterocolitis were investigated with a full sepsis screen and serial abdominal radiographs. Necrotising enterocolitis was diagnosed and staged according to modified Bell's criteria.

Results-Nine of 71 infants receiving oral vancomycin and 19 of 69 infants receiving the placebo solution developed necrotising enterocolitis $(p=0.035)$. Infants with necrotising enterocolitis were associated with a significant increase in mortality $(p=0.026)$ and longer duration of hospital stay $(\mathrm{p}=\mathbf{0 . 0 0 2})$.

wever, widespread implementatic this preventive measure is not recommended, as it would only be effective in necrotising enterocolitis caused by Gram positive organisms and could increase the danger of the emergence of vancomycin resistant or dependent organisms. Its use should be restricted to a high prevalence nursery for a short and well defined period in a selected group of high risk patients. (Arch Dis Child Fetal Neonatal Ed 1998;79:F105-F109)
\end{abstract} Paediatrics, Prince of Wales Hospital, The Chinese University of Hong Kong

Y K Siu

$\mathrm{PC} \mathrm{Ng}$

C H Lee

T F Fok

$\mathrm{KW}$ So

K L Cheung

W Wong

Department of

Microbiology

$S$ C K Fung

A F B Cheng

Department of Mathematics, The

Hong Kong University

of Science and

Technology

M Y Wong

Correspondence to:

Professor PC N

Department of Paediatrics,

Level 6, Clinical Science

Building,

Prince of Wales Hospital,

Shatin, New Territories

Hong Kong

People's Republic of China.

Accepted 15 March 1998

Keywords: necrotising enterocolitis; oral vancomycin; prophylaxis

Necrotising enterocolitis (NEC) is predominantly a disease of premature neonates. It has become the most serious and common gastrointestinal emergency in very low birthweight (VLBW) infants. ${ }^{1-3}$ Although its exact pathophysiology is not fully understood, three essen- tial elements have been implicated in pathogenesis: (1) immature and/or hypoxicischaemic bowel injury, resulting in the loss of the intestinal mucosal barrier integrity; (2) enteral feeds providing food substrates for intraluminal bacterial growth; and (3) translocation of bacteria or their toxic products across the intestinal mucosal barrier. ${ }^{4-6}$

As bacterial translocation is considered to be a graded phenomenon ${ }^{4}$ and only likely to occur if enteric bacteria exceed a critical population level $\left(>10^{9-10} / \mathrm{g}\right.$ of stool in an animal model $),{ }^{7}$ therapeutic approaches to lower the intraluminal bacteria density should theoretically decrease the incidence of NEC. ${ }^{8-11}$ Oral vancomycin has been tried ${ }^{10}{ }^{11}$ because of its activity against coagulase negative staphylococci, Clostridium spp, and Gram positive anaerobes, and also because it does not completely sterilise the bowel which could promote colonisation by unwanted pathogens.

Vancomycin is poorly absorbed and so a high drug concentration can be achieved in the gut lumen with minimal risk of systemic toxicity. ${ }^{10}$ Although we have shown that the use of prophylactic oral vancomycin was associated with a significant reduction in the incidence of NEC, the study was unable confidently to determine whether late introduction of enteral feeding was also a contributory factor. ${ }^{10}$ This prospective randomised trial was undertaken to evaluate the effectiveness of oral vancomycin in the prophylaxis of NEC in a high prevalence neonatal intensive care unit, and the adverse effects of this treatment on individual infants and in the neonatal unit.

\section{Methods}

Neonates were eligible for inclusion in the study if their birthweight was less than $1500 \mathrm{~g}$ and did not have: (1) lethal congenital anomalies; (2) major gastrointestinal abnormalities such as oesophageal atresia, intestinal stenosis or atresia, or Hirchsprung's disease; (3) major gastrointestinal surgery before enteral feeding; and (4) congenital cyanotic heart disease.

Around 70 infants weighing less than $1500 \mathrm{~g}$ are admitted to our neonatal intensive care unit each year. All consecutive admissions were considered for the study if they fulfilled the entry criteria. Eligible infants were randomly assigned by computer at 48 hours of postnatal age to receive either vancomycin or placebo 
treatment. The attending team caring for the infants were unaware of the random assignments.

Infants in the treatment group received oral vancomycin (vancomycin hydrochloride, $\mathrm{Ab}$ bott Laboratories, Chicago, IL, USA) 15 $\mathrm{mg} / \mathrm{kg} /$ dose every 8 hours; those in the placebo group received an equivalent volume of placebo solution. The placebo solution was prepared by diluting one drop of a yellow coloured, sugar free, multivitamin preparation, Vimax (MediPharma Ltd., Tsuen Wan, Hong Kong) into $20 \mathrm{ml}$ of sterile water to simulate the colour of vancomycin. Prophylaxis was begun 24 hours before the start of oral feeds. All infants received a 7 day course of treatment. In infants who developed NEC while receiving the study drug, medication was suspended. The prophylactic drug was not restarted when enteral feeding was introduced after the infant had recovered. During the trial period, all other experimental prophylaxis for NEC, such as oral immunoglobulins or other oral antibiotics, were excluded.

All VLBW infants were started on parenteral nutrition, 6\% TrophAmine (McGaw Inc.,
Irvine, CA, USA) and 20\% Intralipid (Kabi Pharmacia AB, Stockholm, Sweden), on day 3. Oral milk feeds were usually started in the first week of life at the discretion of the attending neonatologist and were given as intermittent boluses via an oro-gastric tube starting at 1 $\mathrm{ml} /$ hour. Oral intake was gradually increased at a rate of $0.5-1 \mathrm{ml} /$ hour/day according to tolerance. Infants were fed mother's milk whenever possible and a number of preterm commercial milk formulas were also used.

All infants were examined at least twice a day and closely observed for gastrointestinal problems including abdominal distension, vomiting, gastric retention, signs of peritonitis and blood in the stools. All suspected cases of NEC were investigated with a full sepsis screen which included cerebrospinal fluid, blood, urine, endotracheal aspirate (intubated infants) and stool (daily sample for three consecutive days) cultures for bacteria and fungi; removal of indwelling umbilical lines; and culture of surgical specimens and specific sites such as peritoneal swab or fluid. Serial abdominal radiographs were routinely performed in these infants and screened for evidence of pneumatosis intestina-

Table 1 Comparison of clinical characteristics and incidence of NEC between oral vancomycin and the placebo groups

\begin{tabular}{|c|c|c|}
\hline Clinical features & Oral vancomycin group $(n=71)$ & Placebo group $(n=69)$ \\
\hline Gestational age (weeks) & $29.6(27.7$ to 31.1$)$ & $28.6(27.0$ to 30.1$)$ \\
\hline Birthweight $(\mathrm{g})$ & $1180(935$ to 1350$)$ & $1170(939$ to 1325$)$ \\
\hline Sex (female:male) & $35(50): 36(50)$ & $29(42): 40(58)$ \\
\hline Inborn:outborn & $68(96): 3(4)$ & $67(97): 2(3)$ \\
\hline Antenatal corticosteroid treatment & $50(70)$ & $45(65)$ \\
\hline \multicolumn{3}{|l|}{ Mode of delivery } \\
\hline Vaginal & $32(45)$ & $26(38)$ \\
\hline Caesarean section & $37(52)$ & $42(61)$ \\
\hline Ventouse & $1(1.5)$ & $1(1)$ \\
\hline Forceps & $1(1.5)$ & $0(0)$ \\
\hline \multicolumn{3}{|l|}{ Apgar scores } \\
\hline $1 \min <3$ & $12(17)$ & $7(10)$ \\
\hline $5 \min <3$ & $1(1)$ & $1(1)$ \\
\hline \multicolumn{3}{|l|}{ First arterial blood gas after delivery } \\
\hline $\mathrm{pH}$ & 7.37 (7.29 to 7.46$)$ & $7.36(7.31$ to 7.43$)$ \\
\hline Base excess & $-2.5(-6.1$ to -1.0$)$ & $-2.6(-5.4$ to -0.6$)$ \\
\hline Temperature on admission $\left({ }^{\circ} \mathrm{C}\right)$ & $36.1(35.8$ to 36.5$)$ & $36.2(35.6$ to 36.7$)$ \\
\hline First venous haematocrit after delivery & $0.52(0.47$ to 0.57$)$ & $0.51(0.46$ to 0.54$)$ \\
\hline Lowest mean arterial blood pressure $(\mathrm{mm} \mathrm{Hg})$ & $28(23$ to 32$)$ & $27(21$ to 30.5$)$ \\
\hline Exchange transfusion & $2(3)$ & $0(0)$ \\
\hline Umbilical arterial catheterisation & $42(59)$ & $38(55)$ \\
\hline Umbilical venous catheterisation & $55(77)$ & $59(86)$ \\
\hline Aminophylline treatment & 49 (69) & $46(67)$ \\
\hline \multicolumn{3}{|l|}{ Respiratory distress syndrome } \\
\hline Stage $0-2$ & $45(63)$ & $51(74)$ \\
\hline Stage $3-4$ & $26(37)$ & $18(26)$ \\
\hline \multicolumn{3}{|l|}{ Periventricular haemorrhage } \\
\hline Stage $<2$ & $63(89)$ & $57(83)$ \\
\hline Stage 3-4 & $8(11)$ & $12(17)$ \\
\hline \multicolumn{3}{|l|}{ Patent ductus arteriosus } \\
\hline Indomethacin closure & $35(49)$ & $37(54)$ \\
\hline Surgical ligation & $3(4)$ & $2(3)$ \\
\hline Age started on enteral feeds (days) & 5 (3 to 9$)$ & $6(5$ to 9$)$ \\
\hline Age received full enteral feeds (days) & $27(20$ to 36$)$ & $28(19$ to 45$)$ \\
\hline \multicolumn{3}{|l|}{ Type of milk feeds } \\
\hline Breast & $12(17)$ & $8(12)$ \\
\hline Formula & $28(39)$ & $33(48)$ \\
\hline Mixed & $31(44)$ & $28(41)$ \\
\hline Incidence of $\mathrm{NEC}^{\star}$ & $9(13)$ & $19(28)$ \\
\hline NEC stages & $25(16$ to 42$)$ & $26(17$ to 33$)$ \\
\hline $0-1$ & $62(87)$ & $50(72)$ \\
\hline 2 & $4(6)$ & $12(17)$ \\
\hline 3 & $5(7)$ & $7(11)$ \\
\hline \multicolumn{3}{|l|}{ Stool culture (fungus: Gram (-): Gram (+)) } \\
\hline Before prophylaxis & $1(1): 2(3): 13(18)$ & $2(3): 6(9): 12(17)$ \\
\hline Immediately after prophylaxis & $8(11): 10(14): 1(1)$ & $9(12): 12(17): 2(3)$ \\
\hline Four weeks after prophylaxis & $4(6): 16(23): 2(3)$ & $1(1): 14(20): 0(0)$ \\
\hline Duration of mechanical ventilation (days) & $6(2$ to 14$)$ & 8 (3 to 24$)$ \\
\hline Duration of $\mathrm{O}_{2}$ dependency (days) & $10(3$ to 29$)$ & $13.5(4.5$ to 50.5$)$ \\
\hline Duration of hospital stay (days) & $84(55$ to 112$)$ & 90.5 (66 to 126$)$ \\
\hline Died & $11(15)$ & $13(19)$ \\
\hline
\end{tabular}

Continuous variables are expressed in median (interquartile ranges) and proportions in number of patients (\%). ${ }^{\star} \mathrm{p}<0.05$. 
Table 2 Comparison of infants with and without necrotising enterocolitis

\begin{tabular}{|c|c|c|}
\hline Clinical features & With NEC $(n=28)$ & Without NEC $(n=112)$ \\
\hline Gestational age (weeks) & $28.1(26.3$ to 30.4$)$ & $29.0(27.7$ to 30.9$)$ \\
\hline Birthweight (g) & 1095 (873 to 1348$)$ & 1195 (960 to 1330$)$ \\
\hline Sex (female:male) & $14(50): 14(50)$ & $50(45): 62(55)$ \\
\hline Inborn:outborn & $26(93): 2(7)$ & $109(97): 3(3)$ \\
\hline Antenatal corticosteroid treatment & $17(61)$ & $78(70)$ \\
\hline \multicolumn{3}{|l|}{ Mode of delivery } \\
\hline Vaginal & $18(64)$ & $43(38)$ \\
\hline Caesarean section & $10(36)$ & $66(59)$ \\
\hline Ventouse & $0(0)$ & $2(2)$ \\
\hline Forceps & $0(0)$ & $1(1)$ \\
\hline \multicolumn{3}{|l|}{ Apgar scores } \\
\hline $1 \min <3$ & $5(18)$ & $14(13)$ \\
\hline $5 \min <3$ & $1(4)$ & $1(1)$ \\
\hline \multicolumn{3}{|l|}{ First arterial blood gas after delivery } \\
\hline $\mathrm{pH}$ & $7.37(7.32$ to 7.45$)$ & $7.36(7.30$ to 7.44$)$ \\
\hline Base excess & $-2.4(-5.0$ to -0.80$)$ & $-2.6(-5.6$ to -0.9$)$ \\
\hline Temperature on admission $\left({ }^{\circ} \mathrm{C}\right)$ & $36.0(35.8$ to 36.8$)$ & $36.2(35.6$ to 36.6$)$ \\
\hline First venous haematocrit after delivery & $0.52(0.47$ to 0.55$)$ & $0.51(0.45$ to 0.57$)$ \\
\hline Lowest mean arterial blood pressure $(\mathrm{mm} \mathrm{Hg})$ & $27.5(22.5$ to 31.5$)$ & 27 (22 to 32$)$ \\
\hline Exchange transfusion & $1(4)$ & $1(1)$ \\
\hline Umbilical arterial catheterisation & $18(64)$ & $62(55)$ \\
\hline Umbilical venous catheterisation & $24(86)$ & $90(80)$ \\
\hline Aminophylline treatment & $20(71)$ & $57(51)$ \\
\hline \multicolumn{3}{|l|}{ Respiratory distress syndrome } \\
\hline Stage $0-2$ & $20(71)$ & $86(77)$ \\
\hline Stage 3-4 & $8(29)$ & $26(23)$ \\
\hline \multicolumn{3}{|l|}{ Periventricular haemorrhage } \\
\hline Stage $<2$ & $26(93)$ & $94(84)$ \\
\hline Stage $3-4$ & $2(7)$ & $18(16)$ \\
\hline \multicolumn{3}{|l|}{ Patent ductus arteriosus } \\
\hline Indomethacin closure & $15(54)$ & $57(51)$ \\
\hline Surgical ligation & $2(7)$ & $3(3)$ \\
\hline Age started on enteral feeds (days) & $6.5(4$ to 14.5$)$ & $6(4$ to 9$)$ \\
\hline Age received full enteral feeds (days) & $36(23$ to 61$)$ & $26(19$ to 37$)$ \\
\hline \multicolumn{3}{|l|}{ Type of milk feeds } \\
\hline Breast & $3(11)$ & $17(15)$ \\
\hline Formula & $11(39)$ & $50(45)$ \\
\hline Mixed & $14(50)$ & $45(40)$ \\
\hline \multicolumn{3}{|l|}{ Stool culture (fungus: Gram (-): Gram $(+)$ ) } \\
\hline Before prophylaxis & $0(0): 0(0): 5(18)$ & $3(3): 8(7): 20(18)$ \\
\hline Immediately after prophylaxis & $4(14): 6(21): 0(0)$ & $13(12): 16(14): 3(3)$ \\
\hline Four weeks after prophylaxis & $2(7): 6(21): 1(4)$ & $3(3): 24(21): 1(1)$ \\
\hline Duration of mechanical ventilation (days) & $9.5(2$ to 31$)$ & $6.5(3$ to 16$)$ \\
\hline Duration of $\mathrm{O}_{2}$ dependency (days) & $16(3.5$ to 47.5$)$ & $10(3$ to 34$)$ \\
\hline Duration of hospital stay (days) ${ }^{\star}$ & $115(90$ to 144$)$ & $81(55$ to 110$)$ \\
\hline Died $^{\star}$ & $9(32)$ & $15(13)$ \\
\hline
\end{tabular}

Continuous variables are expressed in median (interquartile ranges) and proportions in number of patients (\%).

${ }^{\star} \mathrm{p}<0.05$.

lis, portal venous gas, ascites and pneumoperitoneum. The suspected cases were staged according to the modified Bell's criteria ${ }^{12}$ by two of the investigators who had no knowledge of the randomisation assignments. Stages II and III disease were considered diagnostic of NEC.

Stool samples for microbiological culture were collected in all studied infants immediately before treatment, immediately after, and 4 weeks after the completion of the prophylaxis. All specimens were cultured on to a wide range of selective media including: (1) deoxycholate citrate (DC) agar, thiosulphate citrate bile sucrose agar, MacConkey agar and selenite-F enrichment broth (further subcultured onto DC agar after incubation), incubated aerobically at $37^{\circ} \mathrm{C}$ for 18 to 24 hours; (2) blood agar supplemented with vitamin $\mathrm{K} 1$ and cycloserine cefoxitin fructose agar incubated anaerobically at $37^{\circ} \mathrm{C}$ for 48 hours; (3) Skirrow agar incubated under microaerophilic conditions at $42^{\circ} \mathrm{C}$ for 48 hours; and (4) Sabouraud dextrose agar incubated aerobically at $30^{\circ} \mathrm{C}$ for 48 hours.

Stool pathogens including Salmonella spp, Shigella spp, thermophilic Campylobacter spp and Vibrio spp were identified using standard biochemical tests, the API systems (bioMérieux, Marcy-I'Etoile, France) and serological tests where appropriate. Heavy pre- dominant growth or pure growth of aerobes, Clostridium spp, and yeast were recorded. The microbiologists who performed the stool cultures were also unaware of the randomisation assignments.

Our statistics revealed a consistently high prevalence of NEC during the five consecutive years before the study. Most cases occurred in preterm neonates $(>95 \%)$ and the incidence ranged from $18-25 \%$ (mean $21 \%$ ) in VLBW infants. Using the previously detected rate of occurrence and our experience of using prophylactic oral vancomycin, ${ }^{10}$ it was determined that 68 infants would be required in each arm of a randomised controlled study to detect a significant difference at the 5\% level with a power of $80 \%$, if the incidence of NEC was to decrease from $21 \%$ to $5 \%$. Fisher's exact test was used to compare proportions and the Wilcoxon rank sum test for comparison of continuous variables.

This study was approved by the Ethics Committee of the Chinese University of Hong Kong. Written informed consent for participation was obtained from the parents before enrolment.

\section{Results}

One hundred and seventy five VLBW infants were admitted to the neonatal intensive care 
unit between February 1994 and August 1996. Sixteen infants died from extreme prematurity and respiratory diseases before 48 hours of age, 19 parents did not give consent, leaving 140 infants who were successfully recruited to the study.

Seventy one infants received oral vancomycin and 69 received the placebo solution. Table 1 compares the clinical characteristics and incidence of NEC between the two groups. Significantly more infants developed NEC in the placebo group (19 of 69 infants)than in the oral vancomycin group (9 of 71 infants; $p=$ 0.035). All other variables including: gestational age; birthweight; mode of delivery; perinatal asphyxia indices; umbilical catheterisation; drug treatment such as antenatal corticosteroids, aminophylline, and indomethacin; feeding patterns; stool cultures; severity of respiratory distress syndrome and periventricular haemorrhage; duration of mechanical ventilation, oxygen dependency, and hospital stay; and mortality did not differ significantly between the two groups.

A significant decrease in the incidence of stool cultures with pure or heavy predominant growth for Gram positive organisms ( $p<$ 0.001 ) and a significant increase in the incidence of stool cultures for Gram negative organisms $(p<0.05)$ was observed immediately and 4 weeks after treatment with oral vancomycin when compared with the pre-treatment incidence. Similarly, a significant increase in the incidence of stool culture with pure or heavy predominant growth for yeast $(\mathrm{p}<0.05)$ was also detected in infants immediately after oral vancomycin treatment. In contrast, only the incidence of stool culture for Gram positive organisms was significantly decreased after receiving the placebo solution when compared with the pre-treatment incidence $(\mathrm{p}<0.01)$.

Table 2 compares the clinical characteristics between infants with and without NEC. NEC was associated with a significant increase in mortality $(p=0.026)$ and longer duration of hospital stay $(\mathrm{p}=0.002)$ in VLBW infants. Although there was a trend towards longer duration of mechanical ventilation and oxygen dependency in affected infants, neither variable reached significance.

Pathogens were isolated from blood and/or peritoneal specimens in six of nine $(67 \%)$ and nine of $19(47 \%)$ NEC infants treated with oral vancomycin and placebo, respectively. Enterobacter spp (3 cases), methicillin resistant Staphylococcus aureus (MRSA 2 cases), and Serratia spp (1 case) were isolated in the oral vancomycin group. In contrast, coagulase negative staphylococci (4 cases), MRSA (2 cases), Enterobacter spp (1 case), Klebsiella spp (1 case), and Serratia spp (1 case) were isolated in the placebo group.

\section{Discussion}

The most common pathogen for late onset ( $>$ $48 \mathrm{~h}$ of birth) neonatal sepsis is coagulase negative staphylococci ${ }^{13-16}$ which mainly affects infants weighing less than $1500 \mathrm{~g}^{14-16}$ and accounts for over $70 \%$ of all septicaemias in neonatal intensive cares. ${ }^{13} 16$ Furthermore, co- agulase negative staphylococci have been implicated as the single most frequent group of organisms recovered from infants with NEC who died, and from peritoneal specimens taken at the time of surgery. ${ }^{17}{ }^{18}$ In recent years these organisms have also become the commonest group responsible for systemic infection in our unit, and the bacteria most frequently isolated from blood and peritoneal cultures in those infants with NEC ( $>50 \%$ of NEC infants with positive cultures). Oral vancomycin was therefore tried in an attempt to lower the incidence of NEC in the unit.

One of the most important risk factors of NEC is prematurity. Although the infants in the treatment group were on average one week more mature than those in the placebo group, this was not significant. Furthermore, their birthweight were more closely matched and hence, it would be very unlikely that the difference in gestation would have contributed to such a striking difference in incidence between the two groups. More importantly, our results show that prophylactic oral vancomycin conferred protection against NEC in preterm, VLBW infants and was associated with a $50 \%$ reduction in its incidence.

The mechanism is likely to be a decrease in the colonisation and multiplication of Gram positive organisms in the gut lumen which results in the decrease of bacterial translocation or invasion across the immature or injured intestinal mucosa. ${ }^{4}$ Our findings also suggest that infants with NEC had $21 / 2$ times the risk of mortality of infants without NEC (table 2). Furthermore, affected infants required a significantly longer period of hospital stay and also tended to require prolonged mechanical ventilation and oxygen supplementation. These results suggest that the morbidity and mortality were associated with development of long term complications, ${ }^{3}$ such as bronchopulmonary dysplasia, as a consequence of severe and prolonged ventilation during the acute phase of the illness, septicaemia, ${ }^{19}$ cholestatic jaundice and liver failure secondary to prolonged hyperalimentation. ${ }^{19}$

No increase in the incidence of systemic infections caused by fungi or Gram negative organisms was observed, nor were vancomycin resistant or dependent organisms isolated from routine microbiological surveillance or specimens obtained for sepsis screening during the study. No serious adverse effects such as impaired renal function, diarrhoea, or toxic serum drug concentration associated with the use of oral vancomycin were encountered. However, a clinically significant change in the stool flora, with heavy predominant growth of yeast and Gram negative organisms after treatment with oral vancomycin, was worrying, and may indicate the replacement of the normal gut flora by unwanted virulent pathogens.

Although this study supports the prophylactic use of oral vancomycin for prevention of NEC in preterm VLBW infants, we do not recommend widespread implementation of this preventive measure. There are great variations in the pattern of bacterial colonisation and the incidence of NEC among different neonatal 
intensive care units. The risks associated with routine use of oral prophylactic antibiotics probably outweigh the benefits in nurseries with a low incidence or in those colonised predominantly with Gram negative organisms. It is also worrying to note a significant change in the stool flora with heavy predominant growth of yeast and Gram negative organisms after treatment with oral vancomycin. Although the development of organisms resistant to vancomycin was not detected in our study, vancomycin resistant staphylococci, ${ }^{20}$ enterococci, ${ }^{21}$ and vancomycin dependent enterococci ${ }^{22}{ }^{23}$ have been reported. As only five infants in the unit received oral vancomycin at any one time, and the duration of our study was limited to 31 months, the proportion of infants exposed to this treatment was relatively small and the study period might have been too short to allow resistant strains to develop. In our opinion, oral vancomycin treatment should be restricted only to treating an NEC outbreak caused by Gram positive organisms ${ }^{11}$ or use in a high prevalence nursery for a short and well defined period in a selective group of high risk infants. In all circumstances, vigilant microbiological surveillance for vancomycin resistant organisms is mandatory. Until the question of safety can be adequately addressed, the use of oral vancomycin for routine prophylaxis of NEC in VLBW infants should remain experimental.

1 Kliegman RM, Fanaroff AA. Necrotizing enterocolitis. $N$ Engl f Med 1984;310:1093-103.

2 Kosloske AM. Epidemiology of necrotizing enterocolitis. Acta Paediatrica (Suppl) 1994;396:2-7.

3 Albanese CT, Rowe MI. Necrotizing enterocolitis. Semin Pediatr Surg 1995;4:200-6.

4 Deitch EA. Role of bacterial translocation in necrotizing enterocolitis. Acta Paediatrica (Suppl) 1994;396:33-6.
5 Mackendrick W, Caplan MS. Necrotizing enterocolitis: New thoughts about pathogenesis and potential treatNew thoughts about pathogenesis and poten
ments. Pediatr Clin North Am 1993:40:1047-59.

ments. Pediatr Clin North Am 1993:40:1047-59.
6 Willoughby RE Jr, Pickering LK. Necrotizing enterocolitis and infection. Clin Perinatal 1994;21:307-15.

7 Deitch EA. Does the gut protect us or injure us when ill in the ICU. In: Cerra F, ed. Perspectives in critical care. St Louis, Missouri: Quality Medical Publishers, 1988:1-32.

8 Egan EA, Mantilla G, Nelson RM, Eitzman DV. A prospective controlled trial of oral kanamycin in the prevention of neonatal necrotizing enterocolitis. F Pediatr 1976;89:46772 .

9 Grylack LJ, Scanlon JW. Oral gentamicin therapy in the prevention of neonatal necrotizing enterocolitis. Am $\mathcal{F}$ Dis Child 1978;132:1192-4.

$10 \mathrm{Ng}$ PC, Dear PRF, Thomas DFM. Oral vancomycin in prevention of necrotising enterocolitis. Arch Dis Child 1988;63:1390-3.

11 Han VKM, Sayed H, Chance GW, Brabyn DG, Shaheed WA. An outbreak of Clostridium difficile necrotizing enterocolitis: a case for oral vancomycin therapy? Pediatrics 1983;71:935-41.

12 Walsh MC, Kliegmann RM. Treatment based on staging criteria. Pediatr Clin North Am 1986;33:179-201.

13 Ng PC, Thomson M, Dear PRF. Dexamethasone and infection in preterm babies: a controlled study. Arch Dis Child 1990;65:54-8.

14 Beck-Sague CM, Azimi P, Fonseca SN, et al. Bloodstream infection in neonatal intensive care unit patients: results of a multicenter study. Pediatr Infect Dis f 1994;13:1110-6.

15 Khadilkar V, Tudehope D, Fraser S. A prospective study of nosocomial infection in a neonatal intensive care unit. $\mathcal{F}$ Paediatr Child Health 1995;31:387-91.

16 Philip AGS. The changing face of neonatal infection: experience at a regional medical center. Pediatr Infect Dis $\mathcal{f}$ 1994;13:1098-102

17 Mollitt DL, Tepas JJ, Talbert JL. The microbiology of neonatal peritonitis. Arch Surg 1988;123:176-9.

18 Mollitt DL, Tepas JJ, Talbert JL. The role of coagulasenegative staphylococcus in neonatal necrotizing enterocolitis. F Pediatr Surg 1988;23:60-3.

$19 \mathrm{Ng} \mathrm{PC}$, Fok TF, Lee CH, Wong W, Cheung KL. Erythromycin treatment for gastrointestinal dysmotility in preterm infants. $\mathcal{F}$ Paediatr Child Health 1997;33:148-50.

20 Schwalbe RS, Stapleton JT, Gilligan PH. Emergence of vancomycin resistance in coagulase-negative staphylococci. $N$ Engl f Med 1987;316:927-31.

21 Kaplan AH, Gilligan PH, Facklam RR. Recovery of resistant enterococci during vancomycin prophylaxis. $\mathcal{f}$ Clin Microbiol 1988;26:1216-8.

22 Dever LL, Smith SM, Handwerger S, Eng RHK Vancomycin-dependent Enterococcus faecium isolated from stool following oral vancomycin therapy. $₹$ Clin Microbiol 1995;33:2770-3.

23 Farrag N, Eltringham I, Liddy H. Vancomycin-dependent Enterococcus faecalis. Lancet 1996;348:1581-2. 